九州大学学術情報リポジトリ

Kyushu University Institutional Repository

Suppression of mitochondrial transcription initiation complexes changes the balance of replication intermediates of mitochondrial DNA and reduces 7S DNA in cultured human cells 曲，建華

https://doi.org/10.15017/1654748

出版情報 : 九州大学，2015，博士（医学），課程博士 バージョン:

権利関係：やむを得ない事由により本文ファイル非公開（2） 
Regular paper

Molecular Biology

\section{Suppression of mitochondrial transcription initiation complexes changes the balance of replication intermediates of mitochondrial DNA and reduces 7S DNA in cultured human cells}

Jianhua Qu, Takehiro Yasukawa, Dongchon Kang

Department of Clinical Chemistry and Laboratory Medicine, Graduate School of Medical Sciences, Kyushu University, 3-1-1 Maidashi, Higashi-ku, Fukuoka 812-8582, Japan

Running title: Initiation of human mtDNA replication

Correspondence should be addressed to: Takehiro Yasukawa, Department of Clinical Chemistry and Laboratory Medicine, Graduate School of Medical Sciences, Kyushu University, 3-1-1 Maidashi, Higashi-ku, Fukuoka 812-8582, Japan. Tel: +81-92-642-5752, Fax: +81-92-642-5772, email: ytakehir@cclm.med.kyushu-u.ac.jp

Abbreviations: COX, subunits of cytochrome $c$ oxidase (complex IV); ddC, 2',3'-dideoxycytosine; 2D-AGE, 2-dimensional agarose gel electrophoresis; D-loop, displacement loop; GAPDH, glyceraldehyde 3-phosphate dehydrogenase; HSP, heavy strand promoter; H strand, heavy strand; LSP, light strand promoter; L strand, light strand; mtDNA, mitochondrial DNA; NCR, major noncoding region; ND, subunits of NADH-ubiquinone oxidoreductase (complex I); RI, replication intermediate; RITOLS, ribonucleotide incorporation throughout lagging strand; POLRMT, mitochondrial RNA polymerase; TFAM, mitochondrial transcription factor A; TFB2M, mitochondrial transcription factor B2. 
Summary

Analysis of replicating mammalian mitochondrial DNA (mtDNA) suggested that initiation of the replication occurs not only at the specific position, Ori-H but also across a broad zone in mtDNA. We investigated relationship of mitochondrial transcription initiation which takes place upstream of Ori-H and mtDNA replication initiation through analysing the effect of knockdown of mitochondrial transcription factor B2, TFB2M and mitochondrial RNA polymerase, POLRMT, components of the transcription initiation complexes in cultured human cells. Under the conditions where suppression of the transcription initiation complexes was achieved by simultaneous depletion of TFB2M and POLRMT, decrease of replication intermediates of mtDNA RITOLS replication mode accompanied reduction in mtDNA copy number. On the other hand, replication intermediates of coupled leading and lagging strand DNA replication, another proposed replication mode, appeared to be less affected. The findings support the view that the former mode involves transcription from the light strand promoter, and suggest that initiation of the latter mode is independent from the transcription and has distinct regulation. Further, knockdown of TFB2M alone caused significant decrease of 7S DNA, which implies that transcription initiation complexes formed at the light strand promoter engage 7S DNA synthesis more frequently than the initiation of productive replication and transcription.

Keywords: mitochondrial DNA replication, mitochondrial RNA polymerase, mitochondrial transcription factor, 7S DNA, transcription initiation 


\section{Introduction}

Human mitochondria maintain the multicopy circular genome of approximately $16.6 \mathrm{~kb}$, the mitochondrial DNA (mtDNA). It encodes 13 subunits for the oxidative phosphorylation complexes, and 2 ribosomal RNAs (rRNA) and 22 transfer RNAs (tRNAs) for translation of the subunits in mitochondria. All other mitochondrial proteins including those involved in the transcription and replication of mtDNA are encoded on nuclear DNA. mtDNA is highly compact with very little noncoding DNA except for the 1-kb major noncoding region (NCR) flanked by tRNA ${ }^{\text {Phe }}$ and $\mathrm{tRNA}^{\text {Pro }}$ genes from which transcription and many replication events are initiated (l). The two strands of mtDNA are distinguished on the basis of the nucleotide composition and named light strand (L strand) and heavy strand ( $\mathrm{H}$ strand). Mitochondrial transcription occurs polycistronically from the light strand promoter (LSP) and the heavy strand promoters (HSP1 and HSP2) which are located within or at the vicinity of NCR (2), and the polycistronic transcripts are processed to yield mRNA, rRNA and tRNA (3). Human mtDNA has strong coding bias in the two strands; whilst transcription from LSP produces only 1 mRNA and 8 tRNAs, the rest of 12 mRNA and 14 tRNA and both rRNAs are provided by transcription from HSPs. Transcription of mammalian mtDNA is operated by a mitochondria-specific enzyme, mitochondrial RNA polymerase (POLRMT/mtRNAP). It requires two auxiliary factors for the initiation step; mitochondrial transcription factor B2 (TFB2M/mtTFB2) and mitochondrial transcription factor A (TFAM/mtTFA) (2). Sequence of the initiation event was recently proposed $(4,5)$; upon bending of mtDNA induced by the binding of TFAM to a high-affinity site upstream of the transcription start position, POLRMT is recruited, followed by the assembly of TFB2M to form the full initiation complex, and transcription initiation takes place. It was suggested that the presence of TFB2M is critical at the initiation step, but once transcription enters into the elongation step it likely dissociates from the complex and is no longer required for transcription (6).

For three decades mammalian mtDNA had been generally accepted to replicate solely via a strand-displacement model, which entails a long stretch of single-stranded DNA region in mtDNA replication intermediates (RIs) $(7,8)$. A series of recent studies, however, proposed that mammalian mitochondria operate two replication modes, both of which have essentially duplex RIs (9-15). One is 
a novel DNA replication mechanism, designated as RITOLS replication (13): while the nascent $\mathrm{H}$ strand is DNA, processed transcripts are transiently incorporated throughout the lagging strand (15), and they are replaced by DNA only after considerable delay. In addition to RITOLS RIs that are modified extensively by the treatment with RNase H, which degrades RNA in DNA-RNA hybrid, mtDNA RIs consisting of RNase H-resistant, duplex DNA were clearly observed. The presence of such RIs implied the operation of conventional coupled leading and lagging strand DNA synthesis in mammalian mitochondria $(1,12,13)$. Debate continues over the mechanism of mammalian mtDNA replication. RITOLS model proposes that the strand-displacement model was based on the partially single-stranded RIs that were generated as a result of ribonucleotide loss from the RIs of RITOLS replication $(10,13-15)$. On the other hand, occupancy profile of mtDNA by mitochondrial singlestranded DNA binding protein and results from in vitro replication assays were proposed to validate the strand displacement model (16). Also, nucleotide substitution patterns of vertebrate mtDNAs were suggested to be linked with the model (17).

Initiation of $\mathrm{H}$ strand synthesis in RITOLS replication was found to be confined to the NCR and the major initiation position is likely to be the one called Ori- $\mathrm{H}$ or $\mathrm{O}_{\mathrm{H}}(1,13)$. In human mtDNA Ori-H is located $\sim 200$ nucleotide downstream of LSP (18). Many publications pointed to a widelyknown model that transcription from LSP would provide (long) RNA primer for initiation of the nascent H strand DNA synthesis from Ori-H (19). However, it is the very recent report (20) that unambiguously demonstrated the presence of the key molecules which assures the validity of the model; RNA-DNA covalently-attached molecules whose 5' RNA ends are mapped to LSP and the transition site from RNA to DNA is located at Ori-H (and Ori-b). Such molecules were clearly detected upon knockout of RNase H1, the only known RNase H in mammalian mitochondria, in mouse embryonic fibroblasts (MEFs) (20). In contrast to the RITOLS replication mode, initiation of the proposed coupled leading and lagging strand DNA replication mode appears to occur across a broad zone of several kilobases of mtDNA bidirectionally not only in mammals but also in birds $(11,21)$. How this type of replication initiation events are operated and controlled remains obscure. Another notable feature of mammalian mtDNA is the formation of a short triple-stranded region, or displacement loop (D-loop) $(22,23)$, which is located in the NCR. The third strand, 
designated 7S DNA, is about 650 nucleotide long in humans, spanning from Ori-H (8). 7S DNA was proposed to be a primer or an aborted product for $\mathrm{H}$ strand DNA synthesis. A role in the regulation of transcription was also suggested (8). Recently it was proposed that D-loop serves as the binding centre of proteins that regulate dynamics of mitochondrial nucleoids $(24,25)$. However, the relation of 7S DNA, replication and transcription is still ambiguous.

Two preceding reports conducted manipulation of the expression level of TFB2M in cultured cells. HeLa cells that constitutively overexpressed TFB2M had elevated copy number of mtDNA (26). A positive correlation of the increase and decrease of TFB2M levels and mtDNA amount was shown when the levels of TFB2M expression were manipulated in Drosophila cultured cells (27). In consideration with the above reports, crucial temporal participation of TFB2M into the mitochondrial transcription initiation $(6)$ and the recently advanced understanding in the mechanism of mtDNA replication (1), in the current study we purposefully down-regulated the mitochondrial transcription initiation complexes by acutely suppressing not only TFB2M but also POLRMT using siRNA transfection technique in cultured human HeLa cells. We then carefully analysed the effects on the mitochondrial transcripts, mtDNA and 7S DNA and attempted to elucidate the relationships between the RITOLS replication and coupled leading and lagging strand DNA replication as well as between the replication initiation and 7S DNA synthesis and LSP-dependent transcription initiation in human mitochondria. Our data indicate that whilst the activity of the transcription initiation complexes is involved in the initiation of RITOLS replication, the other mode of replication does not appear to be dependent on it, suggesting that the proposed coupled leading and lagging strand DNA replication may have a specific regulation mechanism for the initiation. Further, while simultaneous depletion of TFB2M and POLRMT was required to achieve substantial decrease of mtDNA copy number, knockdown of TFB2M alone caused significant reduction in 7S DNA. The differential effect implies that initiation complexes formed at LSP engage synthesis of 7S DNA more frequently than the initiation of RITOLS replication. 


\section{Materials and methods}

\section{Cell culture, transfection and ddC treatment}

HeLa cells were cultured in "low glucose" DMEM (Sigma D6046) supplemented with 9\% (v/v) FBS and penicillin-streptomycin. Approximately 24 hours before transfection cells were seeded on 6-well plates at 70,000 cells/well. Short interference double-stranded RNA (siRNA) was mixed with INTERFERin (Polyplus-transfection) in Opti-MEM (ThermoFisher Scientific) and the mixture was incubated to form transfection complexes of siRNA and the reagent. During the incubation cells were fed with fresh DMEM (Sigma D6046) and the above mixture was added to the wells to transfect cells with siRNA at a final concentration of $10 \mathrm{nM}$. In case two siRNAs were transfected simultaneously, the concentration of each siRNA was $10 \mathrm{nM}$. Four hours after transfection, the medium was replaced with "high glucose" DMEM (Sigma D6429) supplemented with 9\% (v/v) FBS, $50 \mu \mathrm{g} / \mathrm{ml}$ uridine, 1 $\mathrm{mM}$ pyruvate and penicillin-streptomycin. We intentionally used the "rich medium" to support cells after transfection. Four days after transfection cells were harvested for analyses. For monitoring cell growth cells were seeded to multiple wells of 6-well plates and transfection was performed as above. The number of cells were counted 2, 3 and 4 days after transfection (duplicate per condition) using a Z1 Coulter Particle Counter (Beckman Coulter). The sequences of siRNA used were: TFB2M siRNA-1; 5'-GCU UAG ACU UUA AGC GUU A dTdT -3' (sense) and 5'- UAA CGC UUA AAG UCU AAG C dTdT -3' (antisense), TFB2M siRNA-2; 5'- ACC AAG AAC UUA ACA CCU A dTdT -3' (sense) and 5'- UAG GUG UUA AGU UCU UGG U dTdT -3' (antisense), POLRMT siRNA-2; 5'UGC CAA GCA AGG AGG UCA A dTdT -3' (sense) and 5'- UUG ACC UCC UUG CUU GGC A dTdT -3' (antisense), POLRMT siRNA-3; 5'- CCU UCG AGG CGG UGG AUG U dTdT -3' (sense) and 5'- ACA UCC ACC GCC UCG AAG G dTdT -3' (antisense). Design and synthesis of the above siRNAs were ordered to Bonac Corporation. Negative control siRNA was from the same company.

To deplete mtDNA in cultured HeLa cells mtDNA replication was inhibited using 2',3'dideoxycytosine (ddC). Cells were plated on 6-well plates (70,000 cells/well) and allowed to grow for 
approximately 24 hours. Then medium was replaced with fresh medium containing $5 \mu \mathrm{M}$ ddC to initiate the treatment. After incubation for 4 days cells were harvested for subsequent analyses.

\section{Western blot analysis}

Total cellular lysates for protein analysis was prepared using cell lysis buffer consisting of 20 $\mathrm{mM}$ Tris-HCl (pH 7.2), 150_mM NaCl, 2_mM EDTA, $1 \%$ (v/v) NP-40 and 0.1\% (w/v) SDS. The lysates were then sonicated and centrifuged at 13,000 rpm, after which the supernatant was separated from any debris. Total cellular lysates were electrophoresed in SDS-polyacrylamide gels and blotted onto PVDF membranes (Millipore). Proteins were detected using specific primary antibodies: antiTFB2M (ABGENT, AP10145b), anti-POLRMT (SANTA CRUZ, sc-365082), anti-TFAM and $\beta$ actin (SANTA CRUZ, sc-8432). Secondary antibodies used were HRP conjugates. Immunodetection and densitometric analysis of the protein bands were performed using SuperSignal West Dura Extended Duration Substrate (ThermoFisher Scientific) or other detection kits and ImageQuant LAS4000 machine (GE Healthcare).

\section{DNA preparation}

For preparation of total nucleic acid fractions, cells were lysed with Lysis buffer (75 mM $\mathrm{NaCl}, 50 \mathrm{mM}$ EDTA (pH 8), $10 \mathrm{mM}$ Hepes-NaOH (pH 7.2), $1 \%$ (w/v) SDS and $0.2 \mathrm{mg} / \mathrm{ml}$ proteinase $\mathrm{K})$ and the lysates were incubated at $50^{\circ} \mathrm{C}$ for $30 \mathrm{~min}$. Then the nucleic acids were extracted sequentially with phenol and chloroform/isoamyl-alcohol, followed by 2-propanol precipitation. The pellet was rinsed with 70\% (v/v) ethanol, air-dried and dissolved in $10 \mathrm{mM}$ Hepes- $\mathrm{NaOH}(\mathrm{pH}$ 7.2). Mitochondrial nucleic acid fractions were prepared as follows. Where appropriate the following procedure was performed at $4^{\circ} \mathrm{C}$. Cells were disrupted using a Teflon homogeniser in a 
homogenisation buffer containing $225 \mathrm{mM}$ mannitol, $75 \mathrm{mM}$ sucrose, $10 \mathrm{mM}$ Hepes-NaOH, $2 \mathrm{mM}$ EDTA and 2-mercaptoethanol, and the homogenates were centrifuged at $900 \mathrm{~g}$ to remove nucleus and undisrupted cells. The resultant supernatant was then centrifuged at 12,000 g. The crude mitochondrial pellet was lysed with Lysis buffer and the nucleic acids were obtained as described above.

mtDNA analyses

Quantification of mtDNA content was carried out using a real-time quantitative PCR (rtqPCR) method with SYBR Premix EX Taq II (Til RNaseH Plus) (TaKaRa). Total nucleic acid fractions were used for this assay. The primer set for the amplification of a region in human mtDNA was 5'- CGA TGT TGG ATC AGG ACA TC -3' and 5'- AAG GCG CTT TGT GAA ATA GG -3' and that of hypoxanthine phosphoribosyltransferase 1, used as a nuclear gene standard, was 5'- CCT GGG GAT TCC AAA TAC CT -3' and 5'- GGG CAG AAA AGG TCA TCA AA -3'. The mtDNA content in each sample was normalised to the nuclear gene content.

Southern hybridisation analysis of 7S DNA was carried out with total nucleic acid fractions. Restriction enzyme $P v u \mathrm{II}$ linearised the genome-length mtDNA and the enzymatic digestion released 7S DNA from mtDNA molecules. Heat denaturation of DNA to release of 7S DNA from mtDNA was also performed in a buffer containing 9.5 mM Hepes- $\mathrm{NaOH}(\mathrm{pH} 7.2)$ and $25 \mathrm{mM}$ EDTA (pH 8) at $90{ }^{\circ} \mathrm{C}$ for 3 min and the incubation was terminated by transferring the sample tubes into crushed ice. The processed samples were electrophoresed in $1 \%(\mathrm{w} / \mathrm{v})$ agarose gels, and the gels were blotted onto Zeta-Probe Membranes (Bio-Rad Laboratories), followed by fixation of the blotted DNA by UV cross-linking. Southern hybridisation was performed as described previously (12). A radiolabelled probe was hybridised to the blotted membranes in $0.25 \mathrm{M}$ sodium phosphate (pH 7.2), $7 \%$ (w/v) SDS at $65{ }^{\circ} \mathrm{C}$ overnight, after which the membranes were washed with $150 \mathrm{mM} \mathrm{NaCl}, 15 \mathrm{mM}$ sodium citrate buffer $(\mathrm{pH} 7.0)(1 \times \mathrm{SSC})$ and then $1 \times \mathrm{SSC}$ with $0.1 \%(\mathrm{w} / \mathrm{v}) \mathrm{SDS}$ a few times each. The membranes were exposed to phosphorimaging plates and visualisation of the bands and quantification 
of the band intensity was carried out with a BAS2500 instrument (Fuji Film). DNA probes were radiolabelled with Random Primer DNA Labeling Kit Ver.2 (TaKaTa) and $\left[\alpha-{ }_{-}^{32} \mathrm{P}\right] \mathrm{dCTP}(3000$ $\mathrm{Ci} / \mathrm{mmol}$ ) (PerkinElmer) using a PCR fragment of a mtDNA region spanning from nt 16,341 to 151 . Analysis of mtDNA replication intermediates was performed using 2-dimensional agarose gel electrophoresis (2D-AGE) method with nucleic acid samples purified from mitochondria. mtDNA was digested with restriction enzyme DraI and then precipitated and suspended in $10 \mathrm{mM}$ Hepes$\mathrm{NaOH}$ (pH 7.2). The samples were subjected to $2 \mathrm{D}-\mathrm{AGE}$ essentially as described previously $(9,12)$, followed by Southern hybridisation to visualise and quantify the replication intermediate arcs as described above with a radiolabelled DNA probe which covers a mtDNA region corresponding to nt $12,981-13,384$.

\section{Northern hybridisation}

Total RNA fractions were prepared from cells using RNAiso Plus (TaKaRa) according to the manufacturer's instruction. Denaturation of RNA was performed at $65{ }^{\circ} \mathrm{C}$ for $10 \mathrm{~min}$ and the incubation was terminated by transferring the sample tubes into crushed ice. The samples were electrophoresed in 2\% (w/v) agarose gels with MOPS buffer, and the gels were blotted onto Amersham Hybond $\mathrm{N}^{+}$membranes (GE Healthcare), followed by fixation of the blotted RNA by UV cross-linking. The subsequent procedures were essentially same as those for Southern hybridisation as described above. DNA probes used for detection of mitochondrial transcripts were produced with PCR amplification of the corresponding regions of mtDNA as follows: nt 5,904-7,445 for COX I mRNA, nt 7,586-8,269 for COX II mRNA, nt 4,470-5,511 for ND2 mRNA nt 14,149-14,673 for ND6 mRNA. A DNA probe for glyceraldehyde 3-phosphate dehydrogenase (GAPDH) mRNA detection covered the region between nt 160 and 891 of the mRNA. 


\section{Results and Discussion}

Optimisation of the conditions for suppression of expression of TFB2M and POLRMT using siRNA transfection technique

When the effect of knockdown of a mitochondrial protein is studied under the conditions where substantial number of cells die upon siRNA transfection, cell death could affect the mitochondrial status and such influence may hinder the essential effect of the depletion of the target protein. We first optimized the number of cells which are initially seeded and the procedure of transfection of siRNA (see Materials and methods for details). When seventy thousand HeLa cells were seeded on a well of 6-well plates approximately 24 hours prior to transfection and $10 \mathrm{nM}$ siRNA was transfected, followed by 4 day culture of the cells, few cells were detached from the surface of wells and floated in the medium. Also, cell growth did not appear to be compromised substantially when cells were treated with a single siRNA, TFB2M siRNA-1 (TFB2M-1), TFB2M siRNA-2 (TFB2M-2), POLRMT siRNA-2 (POLRMT-2) or POLRMT siRNA-3 (POLRMT-3), as compared to the growth of cells transfected with negative control siRNA (NC). When not only TFB2M but also POLRMT were depleted with combined transfection of TFB2M siRNA-1 and POLRMT siRNA-3 $(\mathrm{T}+\mathrm{P})$, a modest reduction in cell growth was observed (Fig. 1A). Under these conditions the efficiency of knockdown was evaluated. Figure 1B shows a typical result of western blot analysis of TFB2M and POLRMT in the total cellular lysates 4 days after siRNA transfection. We estimated the reduction in the expression levels of the proteins upon knockdown using serial dilution of the protein lysates from NC-treated cells (Fig. 1B, and Supplementary fig. 1). Judging from the immunoblot bands, approximately $90 \%$ reduction in the levels of TFB2M was achieved by our transfection. Similarly, the levels of POLRMT decreased by approximately $80 \%$, confirming efficient knockdown of the target proteins with minimal effect on cell growth. 
To confirm that the knockdown of TFB2M and POLRMT has 'functional' effect on the mitochondrial transcription, the levels of mitochondrial transcripts in the cells that were treated with siRNA and cultured for 4 days were analysed using Northern hybridisation. To our surprise, although significant decrease of the target proteins was confirmed by western blotting (Fig. 1B), the levels of mitochondrial mRNAs which were transcribed either from LSP (ND6 mRNA) or HSP (ND2 mRNA, COX I mRNA and COX II mRNA) reduced modestly upon single knockdown of either TFB2M or POLRMT (ND, subunits of NADH-ubiquinone oxidoreductase (complex I); COX, subunits of cytochrome c oxidase (complex IV)) (Fig. 2). ND6 mRNA is the only mRNA which is produced with the transcription from LSP. When TFB2M and POLRMT were knocked down simultaneously, all four mRNAs exhibited clear decrease but only roughly in 50\%. These results imply that TFB2M and POLRMT that are available for formation of the mitochondrial initiation complexes exist at a large excess in mitochondria and only when both proteins were depleted, forcible down-regulation of the initiation complexes is sufficient to cause substantial effects, significant decrease of all the transcripts analysed. The gradual effect of the single knockdown and double knockdown observed in the reduction of mRNA amounts could be interpreted that the transcription initiation complexes used for 7S DNA synthesis (see below) was diverted to productive transcription upon decline of the total number of the complexes and thus decrease of mRNAs was preceded by that of 7S DNA. With this scenario it is possible to speculate that the fluctuation of 7S DNA synthesis may play a role in maintaining the amount of transcripts from LSP and HSP. In addition we observed that the stability of TFB2M or POLRMT was not lowered by the severely reduced expression levels of the other (Fig. 1B, Supplementary fig. 1), nor depletion of mtDNA using ddC which is a widely-used inhibitor of mitochondrial DNA polymerase $\gamma$ (12) (Fig. 3), within the duration of the experimental setting in this study. The relative number of TFB2M and POLRMT to mtDNA was estimated to be $\sim 8.6$ and $\sim 6.2$, respectively (26). Knockdown of these proteins in our system probably drove the molar ratio of both TFB2M and POLRMT to mtDNA around 1 according to the quantification of western blotting bands (Supplementary fig. 1). The recent in vitro studies proposed that the bending of mtDNA at the vicinity of the promoter site by TFAM is the crucial step for the assembly of the full mitochondrial 
transcription initiation complexes (POLRMT, TFB2M and TFAM) (4,5). Supplementation to HeLa cells depleted with the endogenous TFAM of the exogenous TFAM lacking the C-terminal 'tail' domain which is necessary for the stimulation of transcription restored mtDNA copy number, but not the transcription activity (28). It is thus possible to assume that if the roles of TFAM in the transcription and mtDNA maintenance are regulated by an unknown mechanism, such as posttranslational modification, the amount of TFAM available for the former role could be a determinant of the transcription initiation activity.

We additionally looked into the relationship of the mitochondrial transcription and the amount of template, mtDNA. Whereas mtDNA copy number was reduced severely to below $10 \%$ to the normal after culturing cells in ddC-containing medium for 4 days (Fig.3B), the levels of mitochondrial transcripts in ddC-treated cells declined modestly ( 40\%) (Supplementary fig. 2). The reason of the results is unclear, but possible interpretation of them could be that the activity of the transcription initiation complexes for the production of RNA was elevated or the mitochondrial transcripts were stabilised to compensate the severe reduction of the template.

mtDNA replication and 7S DNA synthesis were affected differentially by knockdown of TFB2M and POLRMT

We next investigated the involvement of the LSP-dependent transcription in the maintenance of mtDNA under the conditions where the acute down-regulation of the mitochondrial transcription initiation complexes through depleting the core components of the complexes, TFB2M and POLRMT was confirmed to affect the transcript levels significantly. The levels of TFAM were not manipulated as the protein was proposed to be not only a transcription initiation factor but also the major mtDNA packaging/maintenance protein through binding to mtDNA in a sequence non-specific manner $(29,30)$. It was shown that TFAM stabilises mtDNA through the latter function (28). Total nucleic acid fractions were prepared from cells that were treated with siRNAs targeting TFB2M mRNA and POLRMT mRNA and cultured for 4 days. rt-qPCR analysis of mtDNA copy number showed that 
whilst the single knockdown of either TFB2M or POLRMT did not appear to influence the maintenance of mtDNA substantially, the copy number clearly decreased upon simultaneous suppression of the two proteins which most likely generated the conditions of maximal downregulation of the transcription initiation complexes (Fig. 4A). Recently RNA species spanning from LSP to Ori-H which is attached to DNA whose 5'-ends are located at Ori-H was unequivocally detected in MEFs with inducible knockout of RNase H1 (20). Consistent with the report, our results indicate that in living cell (in vivo) LSP-dependent transcription initiated by the mitochondrial transcription initiation complexes is involved in the commencement of mammalian mtDNA replication. In addition, the levels of TFAM declined by approximately $40 \%$ (Fig. 1B and Supplementary fig. 1) when the double knockdown of TFB2M and POLRMT caused reduction in the mtDNA copy number (Fig. 4A). A likely explanation of the results is that TFAM molecules which could not bind to mtDNA were unstable and degraded, as previously proposed (30) and as shown in Figure 3B where the levels of TFAM was lowered by acute depletion of mtDNA.

We then examined 7S DNA upon depletion of TFB2M and POLRMT as the synthesis of 7S DNA is presumed to be primed by RNA transcribed from LSP (19) and thus under control of the activity of the mitochondrial transcription initiation complexes. However, to our knowledge it was not examined before in relation to the activity of the mitochondrial transcription initiation in vivo. Total nucleic acid fractions prepared from cells transfected with siRNA were digested with restriction enzyme $P v u \mathrm{II}$ to produce linearized genome-length mtDNA molecules. Digestion of mtDNA releases 7S DNA from mtDNA. An equal amount of digested samples was electrophoresed in agarose gels and both 7S DNA and genome-length mtDNA were visualised by Southern hybridisation using a radiolabelled probe covering the D-loop region. The data indicate that knockdown of TFB2M alone resulted in an apparent decrease of the levels of 7S DNA and the combined depletion of TFB2M with POLRMT strengthened the effect (Fig. 4B and 4C). Further, taking into consideration of the fact that the copy number of mtDNA decreased in the double knockdown cells, reduction in the absolute amount of 7S DNA in these cells was fairly severe. Essentially same result was obtained when the samples were subjected to heat-denaturation to release 7S DNA (Supplementary fig. 3). Our results thus strongly support the idea that the transcripts from LSP prime 7S DNA synthesis in vivo. 
It is reasonable to presume that the number of the mitochondrial transcription initiation complexes formed at LSP was smaller when both TFB2M and POLRMT were knocked down than when the expression of the former protein alone was suppressed. The difference in the extent of reduction in ND6 mRNA, mtDNA copy number and 7S DNA upon down-regulation of the transcription initiation complexes (Figs. 2 and 4) suggests that the initiation of transcription at LSP is involved in 7S DNA synthesis more frequently than the polycistronic transcription and nascent $\mathrm{H}$ strand synthesis for mtDNA replication.

Knockdown of TFB2M and POLRMT affected the ratio of the mtDNA replication arcs from two replication modes in $2 D-A G E$

Our data so far suggested that LSP-dependent transcription controls DNA synthesis from Ori$\mathrm{H}$, synthesis of the nascent $\mathrm{H}$ strand for productive replication and generation of 7S DNA. RITOLS replication was proposed to initiate within NCR, frequently from Ori-H and proceed effectively undirectionally (13), and the very recent report (20) and the result of this study lend support to the idea that the leading strand (nascent H strand) synthesis of RITOLS replication mode is primed by RNA whose transcription is started from LSP by the action of mitochondrial transcription initiation complexes. We extended our investigation to the bidirectional replication initiation events from the several kilobase region of mtDNA, which was shown in mtDNA of human, mouse, rat and chicken and referred as a broad initiation "zone" (Ori-Z) $(1,11,21)$. Initiation of such replication, which we call Ori-Z initiation here, could take place away from Ori-H. Replication intermediates (RIs) of such replication were found to be duplex DNA, which led to the proposal that the mechanism of the replication is similar to the conventional coupled leading and lagging strand DNA replication $(9,11)$. Two possibilities can be considered for the regulation of Ori-Z initiation. (i) Replication from Ori-Z commences with priming of nascent $\mathrm{H}$ strand synthesis by a long RNA that is transcribed from LSP and the DNA replication occurs bidirectionally, and Ori-Z initiation is under control of LSPdependent transcription. (ii) Melting of double-strand DNA occurs at a given position in Ori-Z and the 
bidirectional replication initiation takes place independently from the transcription from LSP (Supplementary fig. 4).

Using a well-established technique of neutral two-dimensional agarose gel electrophoresis (2D-AGE) $(31,32)$, we investigated the replicating mtDNA molecules to examine the above possibilities. 2D-AGE has been widely used to study DNA replication in different organisms, and was recently applied to mammalian mtDNA replication extensively $(9-15,33)$. As the simultaneous suppression of the expression of TFB2M and POLRMT resulted in the significant reduction in mtDNA copy number (Fig. 4A), we examined the changes in the mtDNA RI arcs upon the double knockdown. Nucleic acids were purified from mitochondria that were prepared from the cells treated with negative control siRNA (NC) and those with both TFB2M siRNA-1 and POLRMT siRNA-3 $(\mathrm{T}+\mathrm{P})$ for 4 days. To preserve mtDNA RIs ribonuclease was not used during the purification procedure. The samples were digested with restriction enzyme DraI and subjected to 2D-AGE. The gels were then blotted onto membranes and mtDNA was visualised by Southern hybridisation using a radiolabelled probe that hybridises to the DraI-digested fragment spanning nt 12,271-16,010 (Supplementary fig. 5). The " $1 \mathrm{~N}$ " spot at the bottom right of the panels was generated from the nonreplicating mtDNA fragments. Besides the $1 \mathrm{~N}$ spot, the $\mathrm{Y}$ arc, the product of duplex DNA RIs derived from the proposed coupled leading and lagging strand DNA replication and slow-moving Y arc (SMY arc), the product of RITOLS replication (see $(1,12)$ for details) were detected (Fig. 5A). The relative intensity of the SMY arc against the Y arc in the negative control siRNA-treated cells seemed to be higher than that in the TFB2M and POLRMT double knockdown cells (Fig. 5A). To confirm the visual inspection, the apex region of the SMY arc (R) and that of $\mathrm{Y} \operatorname{arc}(\mathrm{C})$ in each 2D-AGE panel were quantified by phosphorimaging densitometry as shown in the delineation of the Southern hybridisation image. The intensity value of $\mathrm{R}$ was divided by that of $\mathrm{C}$ and the value of $\mathrm{R} / \mathrm{C}$ from the control sample was expressed as 100 and the relative value of that from the double knockdown sample was calculated. The relative R/C value from cells depleted with both TFB2M and POLRMT was found to reduce by $60 \%$ (Fig. 5B), implying that the frequency of RITOLS replication was substantially down-regulated when the levels of the mitochondrial transcription initiation complexes were maximally suppressed under our experimental conditions. Further, the relative intensity of $\mathrm{Y}$ arc 
against the non-replicating fragments (the intensity value of $\mathrm{C}$ divided by that of $1 \mathrm{~N}$ ) appeared to be comparable between the control and double knockdown cells (Fig. 5C). From this result we assume that the down-regulation of the transcription initiation complexes had little influence on the activity of the proposed coupled leading and lagging strand DNA replication. Overall the data obtained from the 2D-AGE analysis suggested that the above-described possibility (ii) - replication with Ori-Z fires without help of transcription from LSP - is likely. Although the mechanism of the melting of doublestrand DNA for Ori-Z initiation is currently unknown, the priming of the initial leading strand DNA synthesis on both strands has potential candidates to play the role; PrimPol and POLRMT. PrimPol is a primase/polymerase recently identified in human cells and is localised both in nucleus and mitochondria (34). It is possible to speculate that the enzyme can prime the initiation of DNA replication at a given site of mtDNA. POLRMT was shown to have the ability to synthesise short RNA on single-strand DNA template without the aid of TFB2M and TFAM and the synthesis is not dependent on transcription promoter sequence (35).

Our data presented in this study strongly support the idea that both productive replication with RITOLS replication mode and synthesis of 7S DNA involve the action of mitochondrial transcription initiation complexes at LSP. Furthermore this work gives support to the idea that the bidirectional initiation of mtDNA replication occurs across Ori-Z and importantly, suggests that unlike RITOLS replication, the replication from Ori-Z seems unlikely to require transcripts synthesised from LSP for the initiation process but the initiation may be regulated by a different mechanism. The next step to gain a deeper understanding of the initiation of mtDNA replication from Ori-Z is to identify the protein players that are involved in the initiation process. 


\section{Acknowledgements}

We thank Yuichi Matsushima (Kyushu University) for valuable advice and technical assistance.

\section{Funding}

This work was supported in part by Grants-in-Aid for Scientific Research from the Japan Society for the Promotion of Science [grant number 25253041 to D.K. and 90646720 to T.Y.], and supported by Japanese Government (Monbukagakusho: MEXT) Scholarship and a Kyushu University doctoral course scholarship to J.Q. 


\section{REFERENCES}

1. Holt, I.J. and Reyes, A. (2012) Human mitochondrial DNA replication. Cold Spring Harb. Perspect. Biol., 4, a012971.

2. Falkenberg, M., Larsson, N.G. and Gustafsson, C.M. (2007) DNA replication and transcription in mammalian mitochondria. Annu. Rev. Biochem., 76, 679-699.

3. Ojala, D., Montoya, J. and Attardi, G. (1981) tRNA punctuation model of RNA processing in human mitochondria. Nature, 290, 470-474.

4. Morozov, Y.I., Agaronyan, K., Cheung, A.C., Anikin, M., Cramer, P. and Temiakov, D. (2014) A novel intermediate in transcription initiation by human mitochondrial RNA polymerase. Nucleic Acids Res., 42, 3884-3893.

5. Posse, V., Hoberg, E., Dierckx, A., Shahzad, S., Koolmeister, C., Larsson, N.G., Wilhelmsson, L.M., Hallberg, B.M. and Gustafsson, C.M. (2014) The amino terminal extension of mammalian mitochondrial RNA polymerase ensures promoter specific transcription initiation. Nucleic Acids Res., 42, 3638-3647.

6. Sologub, M., Litonin, D., Anikin, M., Mustaev, A. and Temiakov, D. (2009) TFB2 is a transient component of the catalytic site of the human mitochondrial RNA polymerase. Cell, 139, 934-944.

7. Robberson, D.L., Kasamatsu, H. and Vinograd, J. (1972) Replication of mitochondrial DNA. Circular replicative intermediates in mouse L cells. Proc. Natl. Acad. Sci. USA, 69, 737-741.

8. Clayton, D.A. (1982) Replication of animal mitochondrial DNA. Cell, 28, 693-705.

9. Holt, I.J., Lorimer, H.E. and Jacobs, H.T. (2000) Coupled leading- and lagging-strand synthesis of mammalian mitochondrial DNA. Cell, 100, 515-524.

10. Yang, M.Y., Bowmaker, M., Reyes, A., Vergani, L., Angeli, P., Gringeri, E., Jacobs, H.T. and Holt, I.J. (2002) Biased incorporation of ribonucleotides on the mitochondrial L-strand accounts for apparent strand-asymmetric DNA replication. Cell, 111, 495-505. 
11. Bowmaker, M., Yang, M.Y., Yasukawa, T., Reyes, A., Jacobs, H.T., Huberman, J.A. and Holt, I.J. (2003) Mammalian mitochondrial DNA replicates bidirectionally from an initiation zone. J. Biol. Chem., 278, 50961-50969.

12. Yasukawa, T., Yang, M.Y., Jacobs, H.T. and Holt, I.J. (2005) A bidirectional origin of replication maps to the major noncoding region of human mitochondrial DNA. Mol. Cell, 18, 651-662.

13. Yasukawa, T., Reyes, A., Cluett, T.J., Yang, M.Y., Bowmaker, M., Jacobs, H.T. and Holt, I.J. (2006) Replication of vertebrate mitochondrial DNA entails transient ribonucleotide incorporation throughout the lagging strand. EMBO J., 25, 5358-5371.

14. Pohjoismäki, J.L., Holmes, J.B., Wood, S.R., Yang, M.Y., Yasukawa, T., Reyes, A., Bailey, L.J., Cluett, T.J., Goffart, S., Willcox, S. et al. (2010) Mammalian mitochondrial DNA replication intermediates are essentially duplex but contain extensive tracts of RNA/DNA hybrid. J. Mol. Biol., 397, 1144-1155.

15. Reyes, A., Kazak, L., Wood, S.R., Yasukawa, T., Jacobs, H.T. and Holt, I.J. (2013) Mitochondrial DNA replication proceeds via a 'bootlace' mechanism involving the incorporation of processed transcripts. Nucleic Acids Res., 41, 5837-5850.

16. Miralles Fuste, J., Shi, Y., Wanrooij, S., Zhu, X., Jemt, E., Persson, O., Sabouri, N., Gustafsson, C.M. and Falkenberg, M. (2014) In vivo occupancy of mitochondrial singlestranded DNA binding protein supports the strand displacement mode of DNA replication. PLoS Genet., 10, e1004832.

17. Faith, J.J. and Pollock, D.D. (2003) Likelihood analysis of asymmetrical mutation bias gradients in vertebrate mitochondrial genomes. Genetics, 165, 735-745.

18. Chang, D.D. and Clayton, D.A. (1984) Precise identification of individual promoters for transcription of each strand of human mitochondrial DNA. Cell, 36, 635-643.

19. Shadel, G.S. and Clayton, D.A. (1997) Mitochondrial DNA maintenance in vertebrates. Annu. Rev. Biochem., 66, 409-435.

20. Holmes, J.B., Akman, G., Wood, S.R., Sakhuja, K., Cerritelli, S.M., Moss, C., Bowmaker, M.R., Jacobs, H.T., Crouch, R.J. and Holt, I.J. (2015) Primer retention owing to the absence 
of RNase H1 is catastrophic for mitochondrial DNA replication. Proc. Natl. Acad. Sci. USA, 112, 9334-9339.

21. Reyes, A., Yang, M.Y., Bowmaker, M. and Holt, I.J. (2005) Bidirectional replication initiates at sites throughout the mitochondrial genome of birds. J. Biol. Chem., 280, 3242-3250.

22. Arnberg, A., van Bruggen, E.F. and Borst, P. (1971) The presence of DNA molecules with a displacement loop in standard mitochondrial DNA preparations. Biochim. Biophys. Acta., 246, 353-357.

23. Kasamatsu, H., Robberson, D.L. and Vinograd, J. (1971) A novel closed-circular mitochondrial DNA with properties of a replicating intermediate. Proc. Natl. Acad. Sci. USA, 68, 2252-2257.

24. He, J., Mao, C.C., Reyes, A., Sembongi, H., Di Re, M., Granycome, C., Clippingdale, A.B., Fearnley, I.M., Harbour, M., Robinson, A.J. et al. (2007) The AAA+ protein ATAD3 has displacement loop binding properties and is involved in mitochondrial nucleoid organization. J. Cell. Biol., 176, 141-146.

25. Di Re, M., Sembongi, H., He, J., Reyes, A., Yasukawa, T., Martinsson, P., Bailey, L.J., Goffart, S., Boyd-Kirkup, J.D., Wong, T.S. et al. (2009) The accessory subunit of mitochondrial DNA polymerase gamma determines the DNA content of mitochondrial nucleoids in human cultured cells. Nucleic Acids Res., 37, 5701-5713.

26. Cotney, J., Wang, Z. and Shadel, G.S. (2007) Relative abundance of the human mitochondrial transcription system and distinct roles for h-mtTFB1 and h-mtTFB2 in mitochondrial biogenesis and gene expression. Nucleic Acids Res., 35, 4042-4054.

27. Matsushima, Y., Garesse, R. and Kaguni, L.S. (2004) Drosophila mitochondrial transcription factor B2 regulates mitochondrial DNA copy number and transcription in schneider cells. $J$. Biol. Chem., 279, 26900-26905.

28. Kanki, T., Ohgaki, K., Gaspari, M., Gustafsson, C.M., Fukuoh, A., Sasaki, N., Hamasaki, N. and Kang, D. (2004) Architectural role of mitochondrial transcription factor A in maintenance of human mitochondrial DNA. Mol. Cell. Biol., 24, 9823-9834. 
29. Alam, T.I., Kanki, T., Muta, T., Ukaji, K., Abe, Y., Nakayama, H., Takio, K., Hamasaki, N. and Kang, D. (2003) Human mitochondrial DNA is packaged with TFAM. Nucleic Acids Res., 31, 1640-1645.

30. Kang, D., Kim, S.H. and Hamasaki, N. (2007) Mitochondrial transcription factor A (TFAM): roles in maintenance of mtDNA and cellular functions. Mitochondrion, 7, 39-44.

31. Brewer, B.J. and Fangman, W.L. (1987) The localization of replication origins on ARS plasmids in S. cerevisiae. Cell, 51, 463-471.

32. Brewer, B.J. and Fangman, W.L. (1988) A replication fork barrier at the 3 ' end of yeast ribosomal RNA genes. Cell, 55, 637-643.

33. Brown, T.A., Cecconi, C., Tkachuk, A.N., Bustamante, C. and Clayton, D.A. (2005) Replication of mitochondrial DNA occurs by strand displacement with alternative light-strand origins, not via a strand-coupled mechanism. Genes Dev., 19, 2466-2476.

34. Garcia-Gomez, S., Reyes, A., Martinez-Jimenez, M.I., Chocron, E.S., Mouron, S., Terrados, G., Powell, C., Salido, E., Mendez, J., Holt, I.J. et al. (2013) PrimPol, an archaic primase/polymerase operating in human cells. Mol. Cell, 52, 541-553.

35. Wanrooij, S., Fuste, J.M., Farge, G., Shi, Y., Gustafsson, C.M. and Falkenberg, M. (2008) Human mitochondrial RNA polymerase primes lagging-strand DNA synthesis in vitro. Proc. Natl. Acad. Sci. USA, 105, 11122-11127. 


\section{Figure Legends}

Figure 1. Cell growth and levels of TFB2M, POLRMT and TFAM after siRNA knockdown of TFB2M and POLRMT

(A) Cell growth monitoring. Cells were plated to 6-well plates a day before transfection (Day -1). On Day 0 transfection was performed with negative control siRNA (NC), TFB2M-specific siRNAs (TFB2M siRNA-1 [TFB2M-1] or TFB2M siRNA-2 [TFB2M-2]), POLRMT-specific siRNAs (POLRMT siRNA-2 [POLRMT-2] or POLRMT siRNA-3 [POLRMT-3]) or both TFB2M siRNA-1 and POLRMT siRNA-3 (T+P). Cell number in each well was counted 2, 3 and 4 days after the transfection and plotted. Data represent the mean of three independent transfection experiments \pm SEM. (B) Western blot analysis of TFB2M, POLRMT and TFAM in total cellular lysates prepared from cells treated with negative control siRNA (NC) or siRNAs targeting to TFB2M or POLRMT. $\beta$ actin panels are shown as loading control ( $\beta$-actin (1) is for TFB2M and TFAM and $\beta$-actin (2) is for POLRMT). Serial dilution of the lysates from NC-treated cells was also analysed to estimate the extent of depletion of the proteins after knockdown with the corresponding siRNAs.

\section{Figure 2. Northern hybridisation analysis of mitochondrial transcripts upon knockdown of} TFB2M and POLRMT.

Cells were transfected with negative control siRNA (NC), TFB2M siRNA-1 (TFB2M-1), TFB2M siRNA-2 (TFB2M-2), POLRMT siRNA-2 (POLRMT-2), POLRMT siRNA-3 (POLRMT-3) or both TFB2M siRNA-1 and POLRMT siRNA-3 (T+P). Total RNA fractions purified from transfected cells were subjected to Northern hybridisation analysis. (A) Northern hybridisation images of mtDNAencoded mRNAs for ND6, ND2, COX I and COX II. GAPDH mRNA was used as loading control. (B) Quantitative analysis of the mitochondrial transcripts. Band intensities of ND6 mRNA, ND2 
mRNA, COX I mRNA and COX II mRNA were normalised against that of GAPDH mRNA. The relative levels of each (normalised) mRNA in NC-treated sample was expressed as 100 in each experiment and those of other samples were displayed relative to this. The corresponding transcription promoters for the mRNAs are indicated in parentheses. The graphs give the average of the results from four independent transfection experiments with \pm SEM.

Figure 3. Analysis of the levels of the components of mitochondrial transcription initiation complexes after transient depletion of mtDNA with 2',3'-dideoxycytidine

Levels of the mitochondrial proteins were analysed upon acute depletion of mtDNA using 2',3'dideoxycytidine (ddC). (A) Copy number of mtDNA analysed with real-time quantitative PCR to confirm the reduction of mtDNA. mtDNA content was normalised against nuclear gene content in each sample. The relative mtDNA copy number in the untreated sample (Un) was expressed as 100 in each experiment and that of the ddC-treated sample (ddC) was displayed relative to this. Data represent the mean of three independent transfection experiments \pm SEM. (B) Western blot analysis of TFB2M, POLRMT and TFAM in total lysates prepared from cells treated without or with ddC. $\beta$ actin is shown as loading control.

Figure 4. Analyses of mtDNA copy number and 7S DNA amount upon knockdown of TFB2M and POLRMT.

Total nucleic acids from cells cultured for 4 days after transfection with negative control siRNA (NC), TFB2M-specific siRNAs (TFB2M siRNA-1 [TFB2M-1] or TFB2M siRNA-2 [TFB2M-2]), POLRMT-specific siRNAs (POLRMT siRNA-2 [POLRMT-2] or POLRMT siRNA-3 [POLRMT-3]) or both TFB2M siRNA-1 and POLRMT siRNA-3 $(\mathrm{T}+\mathrm{P})$ were subjected to analyses of mtDNA copy number (A) and 7S DNA (B and C). (A) Relative copy number of mtDNA after depletion of TFB2M 
and/or POLRMT was determined with real-time quantitative PCR. mtDNA content was normalised against nuclear gene content in each sample. The relative mtDNA copy number in the NC-treated cells was expressed as 100 in each experiment and those of cells treated with siRNA targeting to TFB2M and/or POLRMT were calculated relative to this. Data represent the mean of four independent transfection experiments \pm SEM. (B) Analysis of 7S DNA. Total nucleic acid fractions were treated with $P v u \mathrm{II}$ and electrophoresed, followed by Southern hybridisation. The bands corresponding to the linearised genome-length mtDNA and 7S DNA are indicated by arrowheads. (C) Quantification of 7S DNA against the genome-length mtDNA. The intensity of the band corresponding to 7S DNA against that to the genome-length mtDNA in lane NC was expressed as 100 and the relative values of 7S DNA against the genome-length mtDNA in each siRNA treatment were shown in the graph. The graph gives the average of the results from four independent transfection experiments with \pm SEM.

Figure 5. Analysis of mtDNA replication intermediates upon combined knockdown of TFB2M and POLRMT with neutral two-dimensional agarose gel electrophoresis.

(A) DraI-digested mtDNA fragments from cells treated with negative control siRNA (NC) or both TFB2M siRNA-1 and POLRMT siRNA-3 (T+P) were separated by neutral two-dimensional agarose gel electrophoresis and visualised with Southern hybridisation. A schematic drawing of the images is also shown. The non-replicating molecules $(1 \mathrm{~N})$ and replication intermediates (Y arc and SMY arcs) are indicated. The apex regions of the Y arc and SMY arc used for the quantification are approximately indicated as grey rectangles and labelled as C and R, respectively. More information on the presentation and interpretation of the arcs is provided in Supplementary figure 5. (B) Quantification of the replication arcs. Intensity values of $C$ and $R$ were quantified and the value of $R$ divided by $\mathrm{C}$, 'R/C' from NC-treated cells was expressed as 100 in each experiment and the relative $\mathrm{R} / \mathrm{C}$ value from the double knockdown cells $(\mathrm{T}+\mathrm{P})$ was calculated. The graph gives the average of the results from three independent transfection experiments \pm SEM. (C) Examination of the relative 
intensity of $\mathrm{Y}$ arc. The intensity value $\mathrm{C}$ divided by that of $1 \mathrm{~N}$ from NC-treated cells was arbitrally set as 100 in each experiment and the relative value of that from the double knockdown cells was calculated. Data represent the mean of three independent transfection experiments \pm SEM. 